\title{
An improved singularity analysis for ASCAT wind quality control: application to low winds
}

\author{
Wenming Lin ${ }^{1}$, Marcos Portabella ${ }^{1}$, Antonio Turiel ${ }^{1}$, Ad Stoffelen $^{2}$, Anton Verhoef $^{2}$ \\ ${ }^{1}$ Institut de Ciències del Mar - CSIC, Pg. Marítim Barceloneta 37-49, 08003 \\ Barcelona, Spain \\ ${ }^{2}$ Royal Netherlands Meteorological Institute (KNMI), Postbus 201, 3730 AE De Bilt, \\ The Netherlands \\ Phone: +34-603299647, Fax: +34-932309555 \\ Email: wenminglin@icm.csic.es
}

\begin{abstract}
Singularity analysis has proven to be a complementary tool to the Advanced Scatterometer (ASCAT) inversion residual (or Maximum Likelihood Estimator) in terms of wind quality control. In this paper, a new implementation scheme of singularity exponent is developed for ASCAT data analysis. It combines the wavelet projections of the gradient measurements of multiple parameters into the analysis, ensuring that the analyzed parameters contribute equally to the final singularity map. Therefore, the underlying geophysical phenomena in the different ASCAT-derived parameters can be effectively revealed simultaneously on a unique map of singularity exponents. The validation using both buoy winds and European Centre for Medium range Weather Forecasting (ECMWF) forecasts wind output shows that the newly derived singularity exponent significantly improves the current ASCAT wind quality control. In particular, poor-quality ASCAT measurements at low wind and
\end{abstract}


high-variability conditions $(w<4 \mathrm{~m} / \mathrm{s})$ can be effectively screened using the new singularity exponent.

Index terms- ASCAT, maximum likelihood estimator (MLE), singularity analysis, quality control, wind variability 


\section{Introduction}

Singularity analysis (SA) derived from the study of turbulent flows has demonstrated to be a valuable approach to analyze and understand many natural phenomena in scale invariant systems, such as the satellite remote sensing maps of sea surface temperature (SST) [1], sea surface salinity (SSS) [2], and sea surface winds [3][4][5], among others. It derives the local singularity exponent (SE) of a geophysical image, which allows to quantitatively interpret the degree of regularity of all the points or pixels in the image. SE can be used to detect not only the existing geophysical structures, characterized as singularity fronts, but also wind retrieval errors. Previous work shows that SA can effectively detect the presence of rain in ASCAT (onboard Metop-A \& Metop-B) retrieved wind fields [3] [4]. More recently, in [5] SA is proved to be a complementary technique to the inversion residual or Maximum Likelihood Estimator (MLE) for both detecting increased wind variability and quality controlling ASCAT-derived winds.

Up to now, singularity maps of the MLE and the ASCAT-retrieved wind components are derived independently at every wind vector cell (WVC). Then, the minimum SE value from the wind speed, wind direction, and MLE is used at each WVC to generate the final singularity map, which is hereinafter referred to as $\mathrm{SE}_{\mathrm{o}}$. By introducing these quality-sensitive parameters into the singularity analysis, one is able to detect increased noise and/or wind discontinuities (divergence or convergence). However, the process of extracting the minimum SE value from a set of independent singularity maps is simple but not physical. Besides, the final SE value may be 
dominated by a certain parameter which has much larger dynamic range than the other parameters in the analysis, resulting in less evident frontal patterns (e.g., when the MLE dominates) or less patchy patterns (e.g., when the wind direction dominates) in the ultimate SA map.

In this paper, an improved singularity analysis is developed to better accommodate ASCAT-derived wind characteristics. Instead of applying wavelet analysis to characterize singularities from each quality-sensitive parameter, it is proposed to combine the wavelet projections of the gradient measurements of each parameter before extracting SE values by the logarithmic operation [6][7]. A set of weighting coefficients are set for each of the used quality-sensitive parameters when summing the wavelet projections of each parameter to get the combined projection. Through this approach, the analyzed parameters almost equally contribute to the SA map. In addition, only one logarithmic operation is required to get the singularity exponent for each WVC, which is more effective than the previous method in terms of numerical calculation.

Previous studies show that the ASCAT wind quality control is generally effective, except for low wind conditions. On the one hand, the geophysical noise, which is dominated by the sub-cell wind variability, increases significantly as the wind speed decreases [8]. On the other hand, the instrument thermal noise becomes non-negligible due to the low signal-to-noise ratio of the scatterometer measurements [9]. Consequently, for wind speeds below $4 \mathrm{~m} / \mathrm{s}$, the ASCAT wind direction skill is poor and the MLE becomes little effective as quality control (QC) indicator [3]. 
Meanwhile, the verification of ASCAT wind quality at low winds is less accurate, due to spatial representativeness errors, i.e., the wind references (e.g., buoy winds and/or model winds) do not well represent the area-mean ASCAT 25-km scale wind vector under increased wind variability conditions. A new quality indicator is thus required for ASCAT low winds.

Section 2 highlights the basics of the traditional SA method, presents the improved SA scheme (with a combined wavelet projection), and summarizes the implementation of the ASCAT QC. In section 3, the new SA method is applied to three years of ASCAT 12.5-km Level 2 (L2) data, and the collocated buoy winds are used to validate its performance as wind quality control and sub-cell wind variability indicator. Since the improvement of the new SA method is remarkable, in section 4 it is particularly tested for low wind conditions $(w<4 \mathrm{~m} / \mathrm{s})$. Finally, the conclusions can be found in section 5 .

\section{Methodology}

\subsection{SA overview}

Following [10][11], the singularity exponent of a two-dimensional signal $s$ at the point $\mathbf{x}$ is derived from the wavelet projection of its gradient, denoted as:

$$
\mathrm{T}_{\psi}\|\nabla s\|(\mathbf{x}, r)=\int \mathrm{d} \mathbf{y}\|\nabla s\|(\mathbf{y}) \frac{1}{r^{2}} \psi\left(\frac{\mathbf{x}-\mathbf{y}}{r}\right)
$$

where $r$ is the scale factor, and $\psi$ is the wavelet function. If the two-dimensional signal contains a singularity exponent $h(\mathbf{x})$ at the point $\mathbf{x}$ according to the function below,

$$
\int \mathrm{d} \mathbf{x}\|\nabla s\|(\mathbf{x})=\alpha(\mathbf{x}) r^{2+h(\mathbf{x})}+o\left(r^{2+h(\mathbf{x})}\right)
$$


then the wavelet projection of Eq. (1) infers the same exponent as [12]:

$$
\mathrm{T}_{\psi}\|\nabla s\|(\mathbf{x}, r)=\alpha_{\psi}(\mathbf{x}) r^{h(\mathbf{x})}+o\left(r^{h(\mathbf{x})}\right)
$$

where $\alpha(\mathbf{x})$ and $\alpha_{\psi}(\mathbf{x})$ are dimensional and signal-dependent amplitude factors, and $o\left(r^{p}\right)$ becomes negligible in comparison with $r^{p}(p=h(\mathbf{x})$, or $h(\mathbf{x})+2)$ when $r$ goes to zero. Since we are mainly interested in the most singular structures of the analyzed signal, rather than the wavelet projections across multiple scales, the following equation is used to estimate the singularity exponents

$$
h(\mathbf{x})=\frac{\log \left[\mathrm{T}_{\psi}\|\nabla s\|(\mathbf{x}, r) / \mathrm{T}_{\psi}^{0}\right]}{\log r_{0}}+o\left(\frac{1}{\log r_{0}}\right)
$$

where $T_{\psi}^{0}$ is the mean value of the wavelet projection over the whole signal. The scale $r_{0}$ is defined as the smallest accessible scale. Negative SE values derived from (4) indicate irregular behavior of the field (higher decorrelation, sharp gradient changes), while the positive values indicate a smooth field over the corresponding points.

In the numerical operation, the wavelet projection at a given point is simply estimated from the vector difference between its actual gradients and those inferred from its four nearest neighbors, which is equivalent to the following function [7],

$$
\mathrm{T}_{\psi}\|\nabla s\|\left(\mathbf{x}, r_{0}\right)=\sqrt{\left(g_{x}^{s}-g_{x 0}^{s}\right)^{2}+\left(g_{y}^{s}-g_{y 0}^{s}\right)^{2}}
$$

where $g_{i}^{s}(i=x, y)$ is the actual gradient of parameter $s$ in the $i$-direction. During the process, $g_{i}^{s}$ at the analyzed point is set to be zero, and then a signal is reconstructed from the gradient series by using the reconstruction operator. Finally, $g_{i 0}^{s}$ (namely the inferred gradient) is derived from the reconstructed signal by applying once more the gradient operator. 


\subsection{Improved $S A$}

Normally, SA is applied on a single image using Eq. (4) in order to estimate the singularity exponents. However, to better understand the underlying geophysical phenomena in a set of images or parameters which are partially correlated with each other, one may want to extract the singularity exponents from more than one image. For the ASCAT application, this was initially carried out in [3], [4] and [5] by applying Eq. (4) to different parameters separately, and then, at every WVC, the minimum $\mathrm{SE}$ value (named as $\mathrm{SE}_{\mathrm{o}}$ ) from the set of derived $\mathrm{SEs}$ was selected to generate the final singularity map. To count the contributions of different parameters to the final singularity map, it is now proposed to accumulate the wavelet projections of all the analyzed images before applying Eq. (4). If the wavelet projection of a single parameter (i.e., the left part of Eq. (3)) is simply denoted as $\mathrm{T}_{\psi}\|\nabla s\|$, the combined projection is written as follows:

$$
\mathrm{T}_{\psi}\|\nabla c\|=\sqrt{\sum_{i} c_{i} \frac{\left(\mathrm{T}_{\psi}\left\|\nabla s_{i}\right\|\right)^{2}}{\left[\sigma\left(\mathrm{T}_{\psi}\left\|\nabla s_{i}\right\|\right)\right]^{2}}}
$$

where $c_{i} \geq 0$ for the weight of the $i^{\text {th }}$ parameter, and $\sigma\left(\mathrm{T}_{\psi}\left\|\nabla s_{i}\right\|\right)$ for the standard deviation (SD) of its wavelet projection. The configuration of $s_{i}$ and $c_{i}$ may be optimized according to different applications. For instance, one should set a relatively high $c_{i}$ value for MLE to better distinguish rain, which usually shows up as blocky structure; or set a relatively high $c_{i}$ value for wind direction in order to better detect the convergent and divergent patterns in the singularity map.

In this paper, the ASCAT wind ambiguity (zonal $u$ and meridional $v$ components), 
which is closest to the background wind, together with the corresponding inversion residual (MLE) are exploited in the new singularity analysis. The objective of this study is to look for an innovative way to account for the contributions of different parameters in singularity analysis, rather than to look for the optimal weight setting. Consequently, $c_{i}=1$ is simply used to balance the contributions of the mentioned parameters in SA, and the explicit expression of Eq. (6) becomes,

$$
\mathrm{T}_{\psi}\|\nabla c\|\left(\mathbf{x}, r_{0}\right)=\sqrt{\frac{\left[\mathrm{T}_{\psi}\|\nabla u\|\left(\mathbf{x}, r_{0}\right)\right]^{2}}{\left[\sigma\left(\mathrm{T}_{\psi}\|\nabla u\|\right)\right]^{2}}+\frac{\left[\mathrm{T}_{\psi}\|\nabla v\|\left(\mathbf{x}, r_{0}\right)\right]^{2}}{\left[\sigma\left(\mathrm{T}_{\psi}\|\nabla v\|\right)\right]^{2}}+\frac{\left[\mathrm{T}_{\psi}\|\nabla \mathrm{MLE}\|\left(\mathbf{x}, r_{0}\right)\right]^{2}}{\left[\sigma\left(\mathrm{T}_{\psi}\|\nabla \mathrm{MLE}\|\right)\right]^{2}}}
$$

Then, $\mathrm{T}_{\psi}\|\nabla s\|$ in Eq. (4) is replaced by the above $\mathrm{T}_{\psi}\|\nabla c\|$ to estimate the singularity exponents from the multiple images. The projections' SD values for $u, v$ and MLE fields estimated from ten days of ASCAT $12.5-\mathrm{km} \mathrm{L2}$ wind data (from June $1^{\text {st }}-10^{\text {th }}$ 2013) are shown as follows,

$$
\begin{cases}\sigma\left(\mathrm{T}_{\psi}\|\nabla u\|\right) & =0.58 \\ \sigma\left(\mathrm{T}_{\psi}\|\nabla v\|\right) & =1.15 \\ \sigma\left(\mathrm{T}_{\psi}\|\nabla \mathrm{MLE}\|\right) & =3.22\end{cases}
$$

The variation of the above $\mathrm{SD}$ values is smaller than $5 \%$ for the $u$ and $v$ wind components, and smaller than $10 \%$ for the MLE field, depending on the time period used, as verified using other randomly selected 10-day periods in year 2011. Therefore, the SD values in (8) are fixed in our SA software for the entire process.

Note that, two ambiguity removal (AR) issues need to be addressed:

- Firstly, for the ASCAT three backscatter measurements (named as triplets) lying close to the surface constructed by the geophysical model function (GMF) [13] [14], the inversion generally leads to two wind solutions or 
ambiguities $180^{\circ}$ apart. However, near the up-, down- and crosswind directions, there is also a substantial number of triplets lying close to GMF surface, but having 3 or 4 solutions. In [15], it is shown that the third and/or fourth solutions are actually an artefact of the inversion procedure and they therefore need to be removed before ambiguity removal.

- Furthermore, the ASCAT Wind Data Processor (AWDP) uses an AR scheme based on data assimilation methods, i.e., the so-called two-dimensional variational ambiguity removal (2DVAR) method [16]. As smaller scales are revealed, the mesoscale analyses and consequently 2DVAR become more challenging. Due to the dual ambiguity nature of the ASCAT wind inversion, it generally leads to two solutions $180^{\circ}$ apart which are equally likely. If the background wind is off by more than $90^{\circ}$ locally, the local ASCAT wind ambiguities actually add information in the analysis step to reinforce the erroneous background. As a result, the front lines resolved by SA likely coincide with those of the background field, rather than with the apparent position depicted by the ancillary parameters, e.g., the inversion residual (namely MLE) or the cloud/rain data from meteorological satellites [17]. Indeed, 2DVAR has been further developed to prevent these locally wrong inferences by applying spatial filtering in order to achieve dynamically consistent wind fields [17]. To mitigate the impact of ambiguity removal errors in the singularity analysis in this study, the wind direction difference between the center WVC and its neighbor WVCs is constrained as follows, 
when calculating the wavelet projection of the gradient of $u / v$ component for the center WVC,

$$
\Delta d^{\prime}=\left\{\begin{array}{cc}
\Delta d & \left(\Delta d \leq 90^{\circ}\right) \\
180^{\circ}-\Delta d & \left(\Delta d>90^{\circ}\right)
\end{array}\right.
$$

where $\Delta d \in\left[0^{\circ}, 180^{\circ}\right]$ means the actual wind direction difference, and $\Delta d^{\prime}$ the constrained result. That is, a direction difference of $180^{\circ}$ (over a sharp convergence or divergence area) is considered as a zero wind direction difference, and in turn, no singularity front (extremely negative SE values) will result in that particular area.

\subsection{Quality control}

Conventionally, the scatterometer wind quality is evaluated using the vector root-mean-square (VRMS) difference between its wind vectors and the reference winds (such as buoy, ECMWF),

$$
\mathrm{VRMS}=\sqrt{\frac{1}{N} \sum_{i}^{N}\left[\left(u_{i}^{\text {scat }}-u_{i}^{r e f}\right)^{2}+\left(v_{i}^{\text {scat }}-v_{i}^{r e f}\right)^{2}\right]}
$$

where $\left(u_{i}^{\text {scat }}, v_{i}^{\text {scat }}\right)$ and $\left(u_{i}^{\text {ref }}, v_{i}^{\text {ref }}\right)$ are the $i^{\text {th }}$ scatterometer and reference wind vector respectively; and $N$ is the total number of collocations.

At first, AWDP uses MLE as a QC indicator. MLE depicts the distance between the measured backscatter triplets and the GMF surface, and also estimates the inconsistency among the triplets. Large MLE value corresponds well to the increased sub-cell wind variability and the decreased wind quality. Operationally, any WVC with MLE $>+18.6$ is flagged as poor wind quality. Afterwards, $\mathrm{SE}_{\mathrm{o}}$ is proposed to complement the MLE-based ASCAT wind QC. SE performs well in detecting the local decorrelation between a WVC and its neighbor cells. Large negative $\mathrm{SE}_{\mathrm{o}}$ value 
corresponds to large spatial decorrelation and inter-WVC wind variability. In [5], the combined $\mathrm{SE}_{\mathrm{o}} / \mathrm{MLE}$ analysis is proposed to improve the MLE-based QC. That is, the VRMS differences between ASCAT and ECMWF winds are studied over a set of 2D bins constructed by SE and MLE; then a 2D flag table is derived using a certain VRMS threshold (in this case $4.5 \mathrm{~m} / \mathrm{s}$ ), i.e., the bins whose VRMS values are higher than $4.5 \mathrm{~m} / \mathrm{s}$ are set for rejection. Furthermore, the measurement variability factor $\left(K_{p}\right)$ [18], which depicts the variability of the backscatter measurements for each antenna beam, is introduced to refine the above $2 \mathrm{D}$ flag table, leading to the development of multi-dimensional histogram (MUDH) QC method.

The 2D or MUDH methods are straightforward but not necessarily effective, since one needs to tune the VRMS threshold carefully, as well as the binning of quality-sensitive parameters. If tuning is not done properly, one of the quality indicators may dominate the vector difference score in a certain 2D or MUDH bin. Subsequently, the data filtered by the other quality indicators in such bin, which may be of relatively good (or poor) quality, are rejected (or kept) unfairly. This often happens when the quality indicators are of distinct characteristics.

Overall, the ASCAT wind quality (or the sub-cell wind variability) is a monotonic function of $\mathrm{SE}$, or $K_{p}$, i.e., the wind quality decreases as $K_{p}$ increases, and $\mathrm{SE}$ decreases. Regarding the signed MLE in [19], the ASCAT wind quality decreases rapidly as $\operatorname{MLE}$ increases for $\operatorname{MLE} \in(0,+\infty)$, while the wind quality degradation is generally small for $\operatorname{MLE} \in(-\infty, 0)$. WVCs with negative MLE values are never rejected according to the current AWDP QC. We are more interested in the positive 
MLE values, where the wind quality is indeed a monotonic function of MLE. Therefore, the following approach is proposed to filter the poor-quality winds [20]:

$$
\mathrm{MLE}>T_{\mathrm{MLE}} \text { or } \mathrm{SE}<T_{\mathrm{SE}} \text { or } K_{p}>T_{K_{p}}
$$

Note that when $T_{x}$ is set to infinity $\left(x=\operatorname{MLE}, K_{p}\right)$ or infinitesimal $(x=\mathrm{SE})$, the corresponding parameter is not used to flag any wind data. Actually, $T_{\mathrm{MLE}}=+18.6$ is used to keep (11) being consistent with the previous MLE(only)-based QC. Moreover, one can achieve equivalent QC effects to the above mentioned 2D or MUDH methods by searching proper SE and $K_{\mathrm{p}}$ thresholds.

\section{Verifications}

\subsection{Data}

In this Section, the new SE derived from (7) (hereinafter referred to as $\mathrm{SE}_{\mathrm{n}}$ ) is compared to both $\mathrm{SE}_{\mathrm{o}}$ and MLE parameters, in terms of $\mathrm{QC}$ and increased wind variability indicators. For such purpose, three years (March 2009- February 2012) of the Ocean and Sea Ice Satellite Application Facility (OSI-SAF) ASCAT 12.5-km L2 data collocated with ECMWF forecasts and moored buoy winds are used. The effective spatial resolution of ASCAT data is determined by the cumulative spatial response function of all the full-resolution backscatter measurements integrated in each WVC [21], which is about twice the WVC size $(25 \mathrm{~km})$. The collocated ECMWF winds, already included in the L2 files, are estimated by interpolating three 3-hourly ECMWF forecasts (selected from $+3 \mathrm{~h}$ to $+18 \mathrm{~h}$ in 3 -h steps) both in space and time to the ASCAT WVC acquisition. Two different moored buoy data sets are used in this study. The first one is retrieved from ECMWF Meteorological Archival 
and Retrieval System (MARS), further referred to as MARS buoys. These buoy winds are segregated into $1 \mathrm{~m} / \mathrm{s}$ speed bins and $10^{\circ}$ direction bins. The collocation criteria for MARS buoy winds are 30 minutes $(30 \mathrm{~min})$ distance in time and $25 \mathrm{~km}$ distance in space from the ASCAT observations. The second one consists of continuous ten-minute (10-min) buoy wind measurements, further referred to as 10-min buoy winds. This buoy data set is available at http://www.pmel.noaa.gov/. The collocation criteria for 10-min buoy winds are $5 \mathrm{~min}$ and $25 \mathrm{~km}$ distance from the ASCAT measurements. Moreover, a series of 25 continuous 10-min buoy measurements are recorded in each collocation.

The total amount of collocations with MARS buoys and with 10-min buoy measurements is about 80,000 and 41,000 , respectively. The former data set is used in section 3.2 and 3.3 to verify the $\mathrm{SE}_{\mathrm{n}}$ impact on wind $\mathrm{QC}$; the latter data set is used in section 3.4 to assess the $\mathrm{SE}_{\mathrm{n}}$ impact on identifying wind variability, and used in section 4 to assess the low winds QC.

\section{$3.2 S E_{n}$ impact on QC effectiveness - one parameter analysis}

The peak of the SE distribution derived from (4) is generally at small positive values, as shown by the histogram of $\mathrm{SE}_{\mathrm{n}}$ in Fig. 1, since the global wind field is expected to be continuous and regular in general, and in math, the wavelet projection's mean value $\mathrm{T}_{\psi}^{0}$ is larger than its median value. However, the peak of the $\mathrm{SE}_{\mathrm{o}}$ distribution is shifted towards small negative values (see the dashed line in Fig. 1), since it is based at each grid point on the minimum SE value from the singularity analysis of the wind speed, wind direction, and MLE (i.e., three different SA maps 
generated by (4) respectively). Hence, by construction, $\mathrm{SE}_{\mathrm{o}}$ values are generally more negative than $\mathrm{SE}_{\mathrm{n}}$ values. To compare the parameter sensitivities to wind quality for different $\mathrm{SE}_{\mathrm{o}}$ and $\mathrm{SE}_{\mathrm{n}}$ distributions, the quality statistics are presented below as a function of the percentage interval sorted by SE values in ascending order. Fig. 2 shows the VRMS difference between ASCAT and buoy winds as a function of the sorted percentage intervals. Lower SE bins correspond to negative SE values, and higher bins to the positive SE values. Each sorted bin contains the same amount of $\left(\mathrm{SE}_{\mathrm{o}}\right.$ and $\left.\mathrm{SE}_{\mathrm{n}}\right)$ data. A zoom of the first $5 \%$ of data (i.e., the data subset with the most variable winds, which also contains poor-quality winds) is also shown at the upper-right corner of each panel, in which marker "I" indicates the uncertainty bar of the estimated VRMS for each bin. Since the operational MLE-based QC rejects approximately $0.3 \%$ of the data for the retrieved winds above $4 \mathrm{~m} / \mathrm{s}$ and almost no rejections are carried out below $4 \mathrm{~m} / \mathrm{s}$ [19], the VRMS for the first $0.3 \%$ of data is shown in Fig. 2(a) (see black square) for comparison purposes. The black square markers in Fig. 2(b-d) indicate the VRMS scores of the operationally rejected WVCs. Note that the VRMS difference between ASCAT and ECMWF winds shows the same trend as that of Fig. 2 (not shown).

As shown in Fig. 2, the VRMS scores for $\mathrm{SE}_{\mathrm{n}}$ are considerably higher than those for $\mathrm{SE}_{\mathrm{o}}$ at the so-called poor-quality or very high wind variability region (leftmost side of the $x$ axis), but lower for the so-called high-quality or stable wind conditions region (right side of the $x$ axis), despite of the small amount of data (i.e., large uncertainty) in the leftmost bins. Therefore, $\mathrm{SE}_{\mathrm{n}}$ is remarkably more effective than $\mathrm{SE}_{\mathrm{o}}$ in 
discriminating the poor-quality WVCs (w.r.t. buoy winds) from the good-quality WVCs over all the wind speed categories. Using the VRMS score of the MLE(only)-based QC as a threshold, the percentage of SE-sorted data with higher VRMS value than the mentioned threshold is computed. For winds above $4 \mathrm{~m} / \mathrm{s}, \mathrm{SE}_{\mathrm{o}}$ detects a slightly larger amount $(0.5 \%)$ of poor-quality winds than MLE, while $\mathrm{SE}_{\mathrm{n}}$ detects a much larger amount of data $(1.1 \%) . \mathrm{SE}_{\mathrm{n}}$ is particularly more effective than $\mathrm{SE}_{\mathrm{o}}$ and MLE at winds below $4 \mathrm{~m} / \mathrm{s}$, indicating that $\mathrm{SE}_{\mathrm{n}}$ may be an effective QC indicator for low winds. This is further tested in Section 4.

\section{$3.3 S E_{n}$ impact on QC effectiveness - combined parameters analysis}

The benefits of $\mathrm{SE}_{\mathrm{n}}$ to ASCAT QC using combined $\mathrm{SE} / \mathrm{MLE}$ or $\mathrm{SE} / \mathrm{MLE} / K_{p}$ analyses are further investigated. According to (11), the collocated ASCAT-MARS buoys data are separately sorted by SE in ascending order, and by $K_{p}$ in descending order at four wind speed categories: $w<4 \mathrm{~m} / \mathrm{s} ; 4 \leq w<7 \mathrm{~m} / \mathrm{s} ; 7 \leq w<10 \mathrm{~m} / \mathrm{s}$; and $w \geq 10 \mathrm{~m} / \mathrm{s}$. Then the sorted data sets are categorized with SE and $K_{\mathrm{p}}$ bins of $0.3 \%$ for the first $5 \%$ of data separately. If any of the binned data is of a VRMS score (ASCAT-MARS buoys) higher than that corresponding to the square maker in Fig. 2, then the retrieved winds in such bin are flagged as poor-quality winds, and therefore rejected. This is carried out in ascending (descending) order for SE $\left(K_{\mathrm{p}}\right)$ until a certain bin for which the VRMS score is lower than that of the square marker in Fig. 2. For that particular bin, a linear interpolation between adjacent bins is carried out to determine the QC threshold (for either SE or $K_{\mathrm{p}}$ ), such that only part of the winds in such bin are flagged as poor-quality winds and the rest flagged as good-quality data. For subsequent bins, the 
ASCAT retrieved winds are flagged as good quality data, and therefore kept. Table 1 summarizes the QC results using different combinations of the quality-sensitive parameters. The above derived bin settings are then tested using the ASCAT-ECMWF collocations in year 2009, and VRMS scores against ECMWF winds are presented in table 2 .

Even though the condition expressed in (11) is not exactly the same as the flag tables used in [5], the multi-parameter $\mathrm{QC}$ results for $\mathrm{SE}_{\mathrm{o}}$ are similar to those presented in [5], indicating that (11) is an effective way for ASCAT wind quality control. Using the $\mathrm{SE}_{\mathrm{n}}$-based methods, the percentages of rejected WVCs are up to four times higher than those of the MLE-based method. Moreover, the rejected WVCs produced with the $\mathrm{SE}_{\mathrm{n}}$-based methods deviate more from both buoy and ECMWF winds than those produced with the MLE(only)-based method.

\subsection{SE $E_{n}$ impact on identifying wind variability}

The assessment and validation of scatterometer wind quality is challenging under increased sub-cell wind variability conditions. Consequently, continuous 10-min buoy wind series are adopted to assess the ASCAT wind quality under such wind conditions [5][20]. The sub-cell variability is depicted by the standard deviation of buoy wind components within a certain measurement period. Note that the SD values of $u$ or $v$ components are particularly combined to express the wind vector variability as

$$
S D_{\text {vector }}=\sqrt{S D_{u}^{2}+S D_{v}^{2}}
$$

The time window for assessing the wind variability is determined by expanding the 10-min-equivalent distance vector in the adjacent time bins (centered at the ASCAT 
measurement time), until the length of the distance vector reaches the WVC size (25 $\mathrm{km})$. That is, for a $5 \mathrm{~m} / \mathrm{s}$ (i.e., $18 \mathrm{~km} / \mathrm{h}$ ) area-mean wind, a $25-\mathrm{km}$ equivalent mean buoy wind is computed by temporally averaging all the buoy data in the range of $+/$ 40 minutes of the ASCAT measurement time. To ensure that the uncertainty in the variability estimation for a single collocation is below 50\%, at least five 10 -min buoy measurements are required in the calculation. Consequently, the minimum period is set to be within \pm 20 min of the ASCAT measurement time. Moreover, the mean buoy wind vector within such period is derived and used as reference in the validation. That is, the mean wind speed is calculated by averaging the wind speed series, and the mean wind direction is derived from the averaged wind $u$ and $v$ components [5].

Table 3 shows the mean SD values of the temporal 10-min buoy wind series for the rejected WVCs by the different QC methods. The same statistics but for stable winds are presented in the last column for comparison. It shows that the $S D_{\text {vector }}$ values for $\mathrm{SE}_{\mathrm{n}}$ are generally higher than those for $\mathrm{SE}_{\mathrm{o}}$, indicating that $\mathrm{SE}_{\mathrm{n}}$ is also more effective than $\mathrm{SE}_{\mathrm{o}}$ in identifying more variable winds, particularly with larger wind direction variability. The VRMS difference between ASCAT and mean buoy winds is shown in table 4. Although the total number of collocations with continuous buoy winds is smaller than that with MARS buoy winds [20], the rejection ratios in table 4 (using continuous winds) are comparable to those in table 1 (using MARS buoy winds). The VRMS scores are in general lower in table 4 than in the last row of table 1, which confirms that the mean buoy winds are more representative of ASCAT area-mean winds than the 10-min buoy winds. In [20], it is shown that when discrepancies 
between ASCAT and mean buoy winds are large, the ASCAT quality (as well as the mean buoy quality) is degraded. The higher VRMS scores associated with $\mathrm{SE}_{\mathrm{n}}$ (as compared to $\mathrm{SE}_{\mathrm{o}}$ ) in the rejected categories of table 4 prove that the $\mathrm{SE}_{\mathrm{n}}$-based $\mathrm{QC}$ is more effective than the $\mathrm{SE}_{0}$-based QC.

Fig. 3 illustrates the vector difference (ASCAT against buoy winds) ratio between QC-rejected and QC-kept winds as a function of the buoy wind vector variability ratio between the mentioned categories. On the one hand, it shows that $\mathrm{SE}_{\mathrm{n}}$ is a better sub-cell wind variability indicator than $\mathrm{SE}_{\mathrm{o}}$ (i.e., $\mathrm{SE}_{\mathrm{n}}$-based methods are located more to the right in the $x$ axis as compared to the other methods). On the other hand, it shows that $\mathrm{SE}_{\mathrm{n}}$-based methods are capable showing larger discrepancies between ASCAT and buoy winds. This is consistent with the first conclusion, i.e., larger discrepancies should occur over higher wind variability areas (i.e., the so-called spatial representativeness). Interestingly, the variance ratio does not change much when VRMS is computed with 10-m buoy winds or mean buoy winds. As suggested by [5], this is an indication of no significant rain contamination over the QC-rejected WVCs. Both ASCAT and buoy winds quality degrade over high wind variability areas [5], but the main cause of this degradation is in both cases the increased wind variability. As clearly seen in the Fig. 3, the use of $\mathrm{SE}_{\mathrm{n}}$ proves to be beneficial for both wind quality control and increased wind variability detection, as the discussion in the previous paragraphs of this section.

\section{Low winds quality control}

At low wind conditions, as shown in [8], the measurement noise substantially increases due to the relatively high sub-cell wind variability, leading to high MLE 
values and poor wind direction skill (the measurements show poor anisotropy). Therefore, the MLE is not an effective quality indicator for $w<4 \mathrm{~m} / \mathrm{s}$, in which condition only $0.2 \%$ of data are rejected with the MLE threshold of +18.6 . Also AWDP incorporates a quality flag defined by $K_{p}$. The operational $K_{p}$ threshold given by (13) is different with the one described in section 3.3,

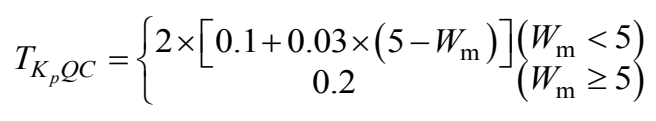

where $W_{\mathrm{m}}$ is the mean wind speed of the retrieved wind ambiguities. For winds above $4 \mathrm{~m} / \mathrm{s}, T_{K_{p} Q C}$ is much higher than the determined $T_{K_{p}}$ (about 0.1 ) in section 3.3, thus the condition (13) only rejects a few number of extreme poor-quality winds. For winds below $4 \mathrm{~m} / \mathrm{s}$, the wind direction variability is relatively high in the rejected data by the operational $K_{p}$-based QC $(1 \%)$, mainly due to the fact that the backscatter measurements are close to the noise floor, and in turn very low winds ( $w<2 \mathrm{~m} / \mathrm{s}$, not shown) are rejected. Since the $\mathrm{SE}_{\mathrm{n}}$ is very sensitive to discrepancies between ASCAT and buoy winds at low winds (see Fig. 2(a)), we further examine different thresholds for QC purposes in this section.

Using the data sorting strategy followed in Fig. 2, Table 5 shows the VRMS difference and the wind speed bias between ASCAT and mean buoy winds for the first $2 \%$, the $2-5 \%$, and the remaining $95 \%$ respectively. The sub-cell variability statistics are shown in the right part of this table. As a comparison, the first row presents the statistics of the data set flagged by the AWDP $K_{p^{-}}$and MLE-based flags. In line with the results for winds above $4 \mathrm{~m} / \mathrm{s}$ presented in Section 3.4, $\mathrm{SE}_{\mathrm{n}}$ proves to be an effective increased wind variability indicator under low wind conditions. As already 
mentioned, [5] and [20] show that under increased wind variability conditions, ASCAT-buoy wind discrepancies increase due to a decrease in both ASCAT and buoy wind quality. Consequently, here we use ASCAT-buoy discrepancies as an ASCAT wind quality indicator, although note that such discrepancies are not an estimation of the absolute ASCAT wind quality but a combination of both ASCAT and buoy wind qualities.

The QC verification at low winds is rather challenging, among others due to the used CMOD5.n GMF generally produces a negative ASCAT wind speed bias w.r.t. the mean buoy winds at low wind conditions (see the last row of Table 5). A new C-band GMF, namely C-2013, has been developed in [22] to address this problem. Using the more stable and good quality winds as reference for the bias correction (i.e., $+0.5 \mathrm{~m} / \mathrm{s}$ ), the flagged data indeed show an increased bias. The quality degradation at low winds may be due to several reasons: on the one hand, the rain splashing on the sea surface and the volume backscatter of raindrops increase the ASCAT backscatter power predominantly at low-wind conditions, thus the retrieved ASCAT winds are apparently higher than the true winds; on the other hand, wind variability could also increase the speed bias, as shown in Fig. 4. Within the same amount of poor-quality data, i.e., $2 \%$ or $2-5 \%$ of data in the second and third rows of Table 5 , the speed bias and most notably the VRMS values associated with $\mathrm{SE}_{\mathrm{n}}$ are higher than those associated with $\mathrm{MLE}$ and $\mathrm{SE}_{\mathrm{o}}$, indicating that $\mathrm{SE}_{\mathrm{n}}$ is more effective in identifying rain-contaminated WVCs at low wind conditions. Comparing to the operational $K_{p}$ and MLE QC flags (the first row of Table 5), $\mathrm{SE}_{\mathrm{n}}$ identifies more poor-quality low 
winds data $(5 \%)$ with higher VRMS and variability scores. In summary, the $\mathrm{SE}_{\mathrm{n}}$ is more effective than MLE, $K_{\mathrm{p}}$, and $\mathrm{SE}_{\mathrm{o}}$ in terms of low-wind quality control. Therefore, it should be used alone for such purpose.

\section{Conclusions}

In this paper, a new singularity exponent based on the exploitation of multiple ASCAT-derived parameters is developed for ASCAT quality-control purposes. It combines the wavelet projections of the gradient measurements of each parameter using a set of weighting coefficients, such that the analyzed parameters contribute more equally to the derived SE map than the previous method in [4]. The newly derived SE is more effective than both the operational and the previous SE-based QC $(w>4 \mathrm{~m} / \mathrm{s})$, i.e., a larger amount of data with larger ASCAT-buoy discrepancies are flagged using either the combined SE/MLE analysis or the MUDH method.

Since $\mathrm{SE}_{\mathrm{n}}$-based method appears to significantly improve the $\mathrm{QC}$ as compared to $\mathrm{SE}_{0}$, the ASCAT wind quality at low wind conditions $(w<4 \mathrm{~m} / \mathrm{s})$ is particularly examined. In spite of the generally high wind variability at low winds, $\mathrm{SE}_{\mathrm{n}}$ is also sensitive to both wind quality and sub-cell wind variability. Therefore, it can also be used for ASCAT QC at low winds.

\section{Acknowledgments}

This work was supported in part by the European Organisation for the Exploitation of Meteorological Satellites Numerical Weather Prediction Satellite Application Facility Associated Scientist project under Reference NWP-AS14-P02 and in part by the Spanish Ministry of Economy and Competitiveness (MINECO) R\&D project under 
Reference AYA2012-39356-C05-03. The ASCAT Level 2 data are provided by EUMETSAT. The software used in this paper was developed through the EUMETSAT NWP SAF. The ECMWF data were retrieved from the ECMWF MARS archive. The buoy rain data are obtained from http://www.pmel.noaa.gov/. We thank Jean-Raymond Bidlot for providing monthly buoy blacklists, and greatly appreciate the anonymous reviewers who helped to improve the quality of this paper.

\section{References}

[1] A. Turiel, V. Nieves, E. Garcia-Ladona, J. Font, M.-H. Rio, and G. Larnicol, "The multifractal structure of satellite sea surface temperature maps can be used to obtain global maps of streamlines," Ocean Sci., 5, 447-460, 2009.

[2] N. Hoareau, M. Umbert, J. Martínez, A. Turiel, and J. Ballabrera-Poy, "On the potential of data assimilation to generate SMOS-Level 4 maps of sea surface salinity," Remote Sensing of Environment, 146, 188-200, 2014.

[3] M. Portabella, A. Stoffelen, W. Lin, A. Turiel, A. Verhoef, J. Verspeek, and J. Ballabrera, "Rain effects on ASCAT wind retrieval: Towards an improved quality control," IEEE Trans. Geosci. Remote. Sens., vol. 50, no. 7, pp. 2495-2506, 2012.

[4] W. Lin, M. Portabella, A. Stoffelen, A. Turiel, and A. Verhoef, "Rain identification in ASCAT winds using singularity analysis," IEEE Trans. Geosci. Remote. Sens. Lett., 11(9), 1519-1523, 2014.

[5] W. Lin, M. Portabella, A. Stoffelen, A. Verhoef, and A. Turiel, "ASCAT wind quality control near rain,” IEEE Trans. Geosci. Remote Sens., vol.53, no.8, pp:4165-4177, 2015.

[6] S. Mallat and W. L. Huang, "Singularity detection and processing with wavelets," IEEE Trans. Inf. Theory, 38:617-643, 1992.

[7] O. Pont, A. Turiel, and H. Yahia, "An optimized algorithm for the evaluation of local singularity exponents in digital signals," in Proc. $14^{\text {th }}$ IWCIA LNCS, J. Aggarwal, R. Barneva, V. Brimkov, K. Koroutchev, and E. Korutcheva, Eds., Madrid, Spain, 2011, pp. 346-357. 
[8] M. Portabella and A. Stoffelen, "Scatterometer backscatter uncertainty due to wind variability,” IEEE Trans. Geosci. Remote. Sens., Vol.44, no.11, pp:3356-3362, 2006.

[9] J. Figa-Saldaña, J.J.W. Wilson, E. Attema, R. Gelsthorpe, M.R. Drinkwater, and A. Stoffelen, "The advanced scatterometer (ASCAT) on the meteorological operational (MetOp) platform: A follow on for European wind scatterometers," Can. J. Remote Sensing, vol. 28, no.3, pp: 404-412, 2002.

[10] A. Turiel, H. Yahia, and C. Pérez-Vicente, "Microcanonical multifractal formalism: a geometrical approach to multifractal systems. Part I: Singularity analysis," Journal of Physics $A, 41: 015501,2008$.

[11] A. Turiel and N. Parga, "The multi-fractal structure of contrast changes in natural images: from sharp edges to softer textures," Neural Comput., vol. 12, pp. 763-793, 2000.

[12] S. Mallat and S. Zhong, "Wavelet transform maxima and multiscale edges," in Wavelets and Their Applications, M. B. Ruskai et al., Eds. Boston, MA: Jones and Bartlett, 1991.

[13] A. Stoffelen and D. Anderson, "Scatterometer data interpretation: measurement space and inversion," J. Atmos. Ocean. Technol., 14, 1298-1313, 1997.

[14] H. Hersbach, "Comparison of C-band scatterometer CMOD5.n equivalent neutral winds with ECMWF,” J. Atmos. Ocean. Technol., 27, 721-736, 2007.

[15] W. Lin, M. Portabella, A. Stoffelen, and A. Verhoef, "On the characteristics of ASCAT wind direction ambiguities," Atmos. Meas. Tech., 6, 1053-1060, 2013.

[16] A. Stoffelen and D. Anderson, "Ambiguity removal and assimilation of scatterometer data," Q.J.R. Meteorol. Soc., 123, pp. 491-518, 1997.

[17] W. Lin, M. Portabella, J. Vogelzang, A. Stoffelen, and A. Verhoef, "On mesoscale analysis and ASCAT ambiguity removal," EUMETSAT, Darmstadt, Germany, NWP SAF Visiting Scientist Reports NWPSAF-KN-VS-014 v.1, August 2015. http://nwpsaf.eu/vs.html

[18] C. Anderson, H. Bonekamp, C. Duff, J. Figa-Saldaña, and J.J.W. Wilson, "Analysis of ASCAT ocean backscatter measurement noise," IEEE Trans. Geosci. Remote. Sens., vol. 50, no. 7, pp.2449-2457, 2012.

[19] M. Portabella, A. Stoffelen, A. Verhoef, and J. Verspeek, "A new method for improving scatterometer wind quality control," IEEE Trans. Geosci. Remote. Sens. Lett., 9(4), 579-583, 2012. 
[20] W. Lin, M. Portabella, A. Stoffelen, J. Vogelzang, A. Verhoef, "ASCAT wind quality control under high sub-cell wind variability conditions," J. Geophys. Res. Oceans, 120(8), pp: 5804-5819, 2015.

[21] R. Lindsley, C. Anderson, J. Figa-Saldaña, and D. Long, "ASCAT spatial response function and land contamination estimation," paper presented at ASCAT High Resolution Processing Workshop, EUMETSAT, Darmstadt, Germany, 2014.

[22] L. Ricciardulli and F. Wentz, "Development of consistent geophysical model functions for different scatterometer missions: Ku and C-band," at the 2012 International Ocean Vector Wind Science Team (IOVWST) meeting, Utrecht, Netherlands, June 2012. https://coaps.fsu.edu/scatterometry/meeting/past.php 


\section{Tables}

Table-1 Percentage and vector root-mean-square (VRMS) difference $[\mathrm{m} / \mathrm{s}]$ between ASCAT and MARS buoy winds using different QC methods. R indicates QC-rejected category, $\mathrm{K}$ represents QC-kept category, and R\% means the QC rejection ratio. (Number of collocations $N=60,536)$

\begin{tabular}{|c|c|c|c|c|c|c||c|c|c|c|c|c|}
\hline \multirow{2}{*}{$w(\mathrm{~m} / \mathrm{s})$} & \multicolumn{3}{|c|}{$\mathrm{SE}_{\mathrm{o}} / \mathrm{MLE}$} & \multicolumn{4}{c||}{$\mathrm{SE}_{\mathrm{n}} / \mathrm{MLE}$} & \multicolumn{3}{|c|}{$\mathrm{SE}_{\mathrm{o}} / \mathrm{MLE} / K_{\mathrm{p}}$} & \multicolumn{3}{|c|}{$\mathrm{SE} / \mathrm{MLE} / K_{\mathrm{p}}$} \\
\cline { 2 - 13 } & $\mathrm{R}$ & $\mathrm{K}$ & $\mathrm{R} \%$ & $\mathrm{R}$ & $\mathrm{K}$ & $\mathrm{R} \%$ & $\mathrm{R}$ & $\mathrm{K}$ & $\mathrm{R} \%$ & $\mathrm{R}$ & $\mathrm{K}$ & $\mathrm{R} \%$ \\
\hline$[4,7)$ & 5.16 & 2.04 & 0.64 & 5.53 & 1.99 & 1.27 & 5.16 & 2.02 & 0.98 & 5.38 & 1.98 & 1.56 \\
\hline$[7,10)$ & 6.63 & 2.02 & 0.98 & 7.24 & 1.97 & 1.23 & 6.63 & 1.98 & 1.35 & 7.00 & 1.95 & 1.54 \\
\hline$\geq 10$ & 9.79 & 2.69 & 0.52 & 11.90 & 2.60 & 0.69 & 10.03 & 2.62 & 0.86 & 11.2 & 2.56 & 0.95 \\
\hline \hline$\geq 4$ & 6.78 & 2.20 & 0.74 & 7.39 & 2.14 & 1.13 & 6.89 & 2.16 & 1.09 & 7.16 & 2.11 & 1.41 \\
\hline
\end{tabular}

Table-2 Percentage and VRMS difference between ASCAT and ECMWF winds using different QC methods. Winds above $4 \mathrm{~m} / \mathrm{s}$ are studied. (Number of collocations $N=17.6$ million)

\begin{tabular}{|c|c||c|c||c|c|}
\hline & MLE-only & $\mathrm{SE}_{\mathrm{o}} / \mathrm{MLE}$ & $\mathrm{SE}_{\mathrm{n}} / \mathrm{MLE}$ & $\mathrm{SE}_{\mathrm{o}} / \mathrm{MLE} / K_{\mathrm{p}}$ & $\mathrm{SE}_{\mathrm{n}} / \mathrm{MLE} / K_{\mathrm{p}}$ \\
\hline VRMS- rejected (m/s) & 6.12 & 6.03 & 6.47 & 5.92 & 6.27 \\
\hline VRMS- kept (m/s) & 2.28 & 2.26 & 2.22 & 2.25 & 2.21 \\
\hline Rejection ratio (\%) & 0.30 & 0.63 & 1.10 & 0.85 & 1.20 \\
\hline
\end{tabular}

Table-3: The mean standard deviation (SD) values of the continuous buoy wind speed, direction and wind components for different QC methods. The last column presents the statistics of stable wind condition (total number $N=28,111$ )

\begin{tabular}{|c|c||c|c||c|c||c|}
\hline & MLE-only & $\mathrm{SE}_{\mathrm{o}} / \mathrm{MLE}$ & $\mathrm{SE}_{\mathrm{n}} / \mathrm{MLE}$ & $\mathrm{SE}_{\mathrm{o}} / \mathrm{MLE} / K_{\mathrm{p}}$ & $\mathrm{SE}_{\mathrm{n}} / \mathrm{MLE} / K_{\mathrm{p}}$ & $|\mathrm{MLE}|<0.5$, \\
$\mathrm{SE}>0$ \\
\hline $\mathrm{SD}($ speed, $\mathrm{m} / \mathrm{s})$ & 1.21 & 1.27 & 1.24 & 1.31 & 1.24 & 0.39 \\
\hline $\mathrm{SD}\left(\right.$ direction, $\left.{ }^{\circ}\right)$ & 18.0 & 18.4 & 22.6 & 20.4 & 22.1 & 4.5 \\
\hline $\mathrm{SD}(u, \mathrm{~m} / \mathrm{s})$ & 1.51 & 1.60 & 1.67 & 1.58 & 1.60 & 0.47 \\
\hline $\mathrm{SD}(v, \mathrm{~m} / \mathrm{s})$ & 1.59 & 1.56 & 1.68 & 1.71 & 1.70 & 0.54 \\
\hline
\end{tabular}


Table-4 VRMS difference between ASCAT and mean buoy winds using different QC methods.

Winds above $4 \mathrm{~m} / \mathrm{s}$ are studied. $(N=28,111)$

\begin{tabular}{|l|l|l|l|l|l|}
\hline & MLE-only & $\mathrm{SE}_{\mathrm{o}} / \mathrm{MLE}$ & $\mathrm{SE}_{\mathrm{n}} / \mathrm{MLE}$ & $\mathrm{SE}_{\mathrm{o}} / \mathrm{MLE} / K_{\mathrm{p}}$ & $\mathrm{SE}_{\mathrm{n}} / \mathrm{MLE} / K_{\mathrm{p}}$ \\
\hline Rejected & 5.53 & 6.20 & 6.90 & 6.17 & 6.50 \\
\hline Kept & 1.91 & 1.86 & 1.81 & 1.83 & 1.79 \\
\hline Rejection ratio & $0.29 \%$ & $0.71 \%$ & $0.99 \%$ & $1.07 \%$ & $1.28 \%$ \\
\hline
\end{tabular}

Table-5: Statistics of the ASCAT wind quality and the sub-cell wind variability at low-wind conditions $(N=7,175)$

\begin{tabular}{|c|c|c|c|c|c|c|c|}
\hline \multirow[t]{2}{*}{ Data set } & & \multicolumn{2}{|c|}{$\begin{array}{l}\text { Wind quality w.r.t. the } \\
\text { mean buoy winds }\end{array}$} & \multicolumn{4}{|c|}{$\begin{array}{l}\text { Sub-cell wind variability, mean SD of temporal buoy } \\
\text { wind series: }\end{array}$} \\
\hline & & VRMS & Speed bias & (speed, $\mathrm{m} / \mathrm{s}$ ) & $\left(\right.$ direction, $\left.{ }^{\circ}\right)$ & $(u, \mathrm{~m} / \mathrm{s})$ & $(v, \mathrm{~m} / \mathrm{s})$ \\
\hline $1.2 \%$ & $\begin{array}{l}\text { AWDP } \\
\text { flags }\end{array}$ & 2.70 & -0.54 & 0.65 & 38.4 & 0.69 & 0.79 \\
\hline \multirow{3}{*}{$\begin{array}{c}\text { Top } 2 \% \\
\text { (Poor-quality) }\end{array}$} & MLE & 3.67 & -0.14 & 0.83 & 21.9 & 0.97 & 0.98 \\
\hline & $\mathrm{SE}_{\mathrm{o}}$ & 3.10 & -0.23 & 0.88 & 29.9 & 1.02 & 1.03 \\
\hline & $\mathrm{SE}_{\mathrm{n}}$ & 4.32 & 0.28 & 0.98 & 26.1 & 1.18 & 1.17 \\
\hline \multirow{3}{*}{$\begin{array}{l}\text { Top 2-5\% } \\
\text { (Poor-quality) }\end{array}$} & MLE & 2.93 & -0.24 & 0.66 & 17.6 & 0.81 & 0.77 \\
\hline & $\mathrm{SE}_{\mathrm{o}}$ & 2.82 & -0.45 & 0.75 & 24.7 & 0.83 & 0.86 \\
\hline & $\mathrm{SE}_{\mathrm{n}}$ & 3.75 & 0.13 & 0.91 & 24.5 & 1.01 & 1.05 \\
\hline $\begin{array}{l}\text { Rest 95\% } \\
\text { (Good-quality) }\end{array}$ & $\mathrm{SE}_{\mathrm{n}}$ & 1.94 & -0.50 & 0.62 & 19.6 & 0.69 & 0.72 \\
\hline
\end{tabular}




\section{Figures}

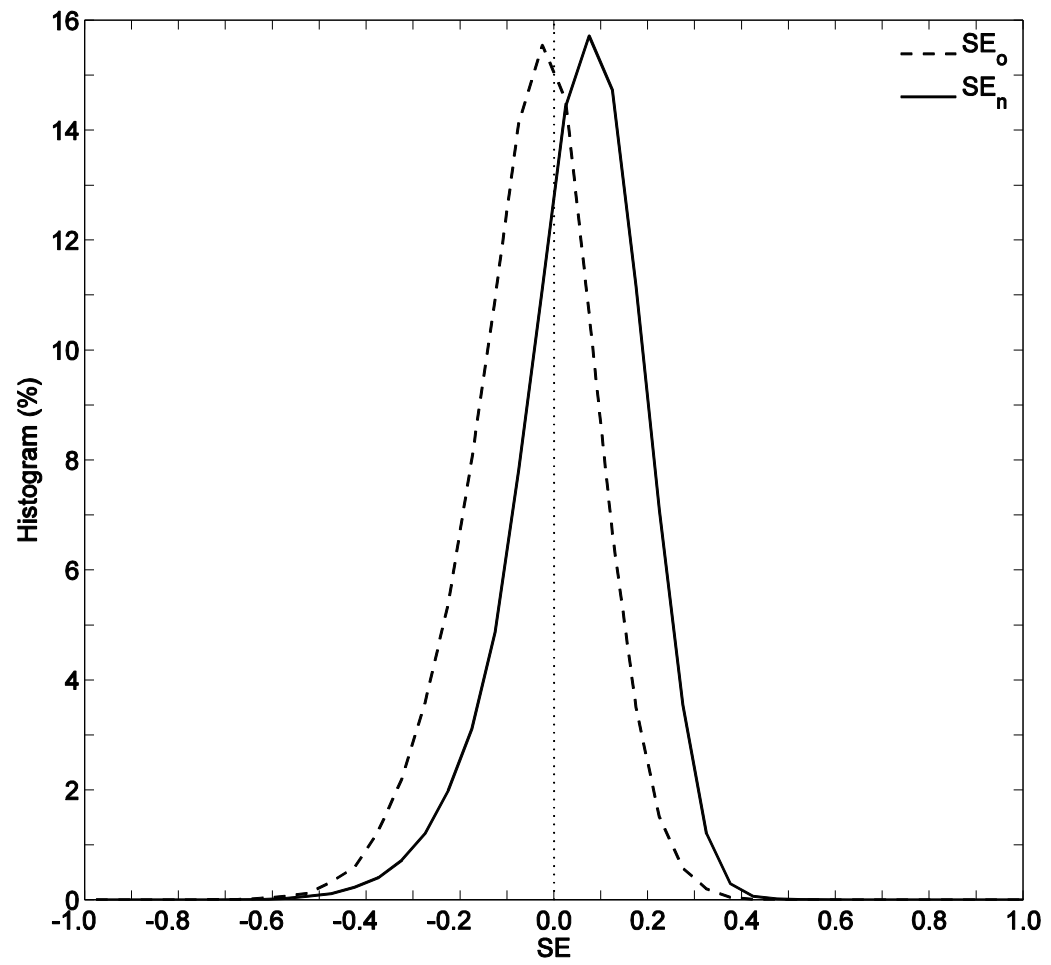

Fig. 1 The histogram of old $\left(\mathrm{SE}_{\mathrm{o}}\right.$, dashed line) and new ( $\mathrm{SE}_{\mathrm{n}}$, solid line) singularity exponents with binning width of 0.05 . 


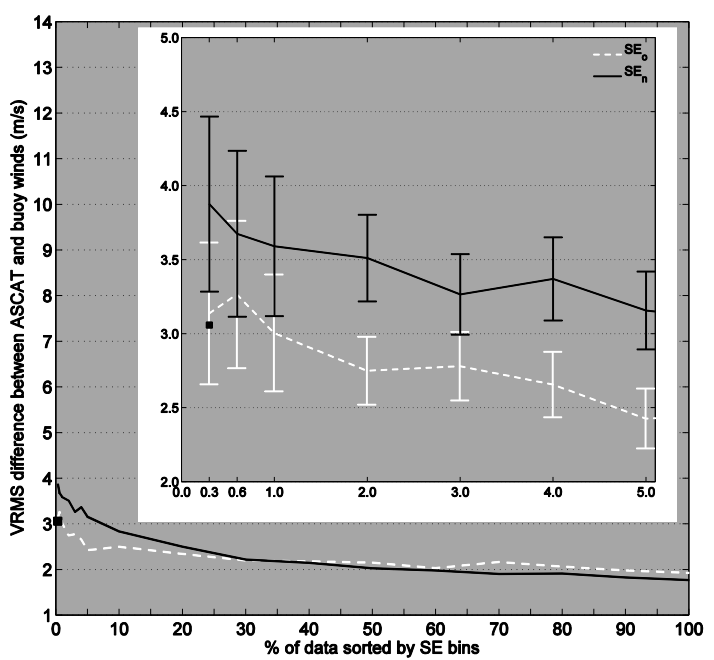

(a)

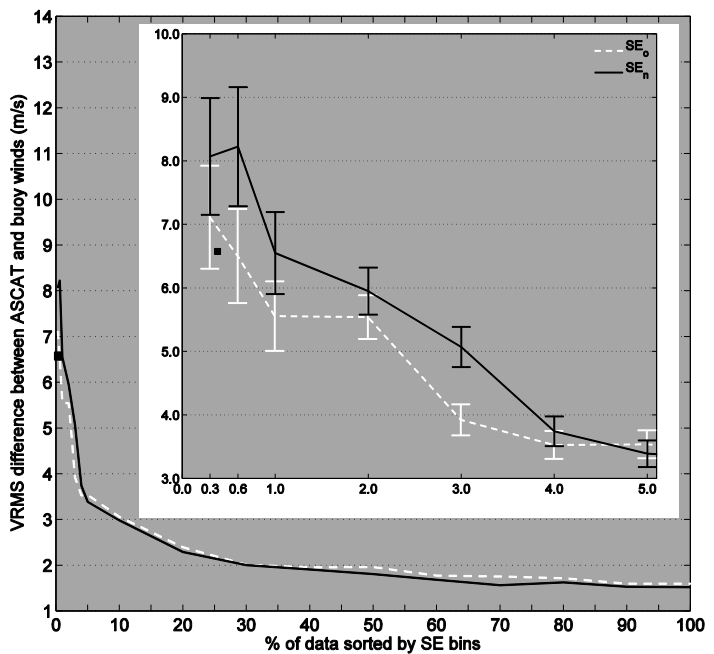

(c)

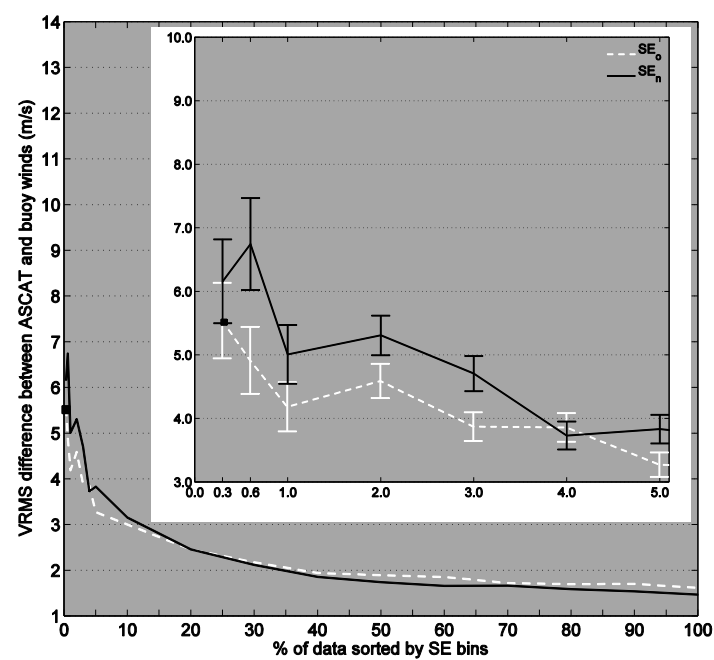

(b)

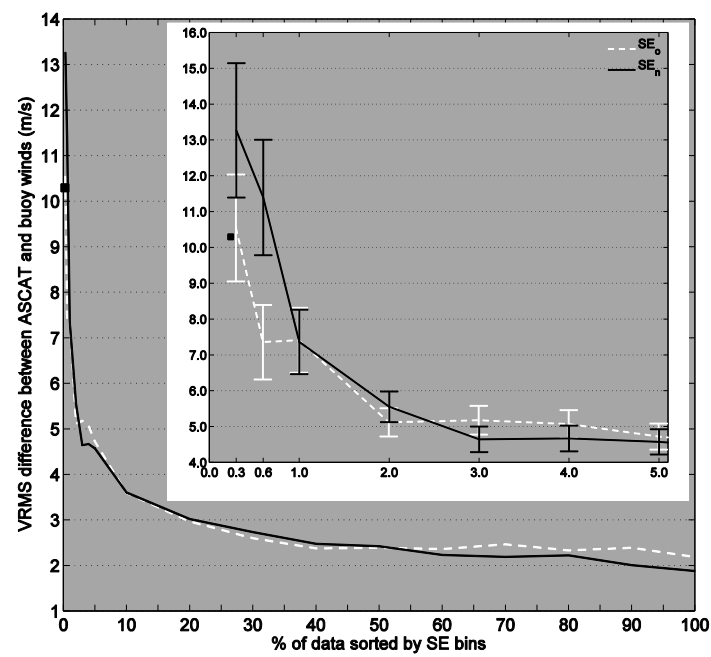

(d)

Fig. 2 VRMS difference between ASCAT and MARS buoy as a function of the sorted percentage intervals at $(a) w<4 \mathrm{~m} / \mathrm{s} ;(b) 4 \leqslant w<7 \mathrm{~m} / \mathrm{s} ;(c) 7 \leqslant w<10 \mathrm{~m} / \mathrm{s} ;$ and, $(d) w \geqslant 10 \mathrm{~m} / \mathrm{s}$. A zoom of the first $5 \%$ of data is shown on the right-upper corner of each panel, in which marker "I" denotes the uncertainty bar of the estimated VRMS for each bin. The square marker in $(a)$ denotes the VRMS score (y-axis) of the top $0.3 \%$ of data; while in $(b)-(d)$ indicates the VRMS score (y-axis) and the rejection ratio ( $x$-axis) of the operational MLE-based QC. 


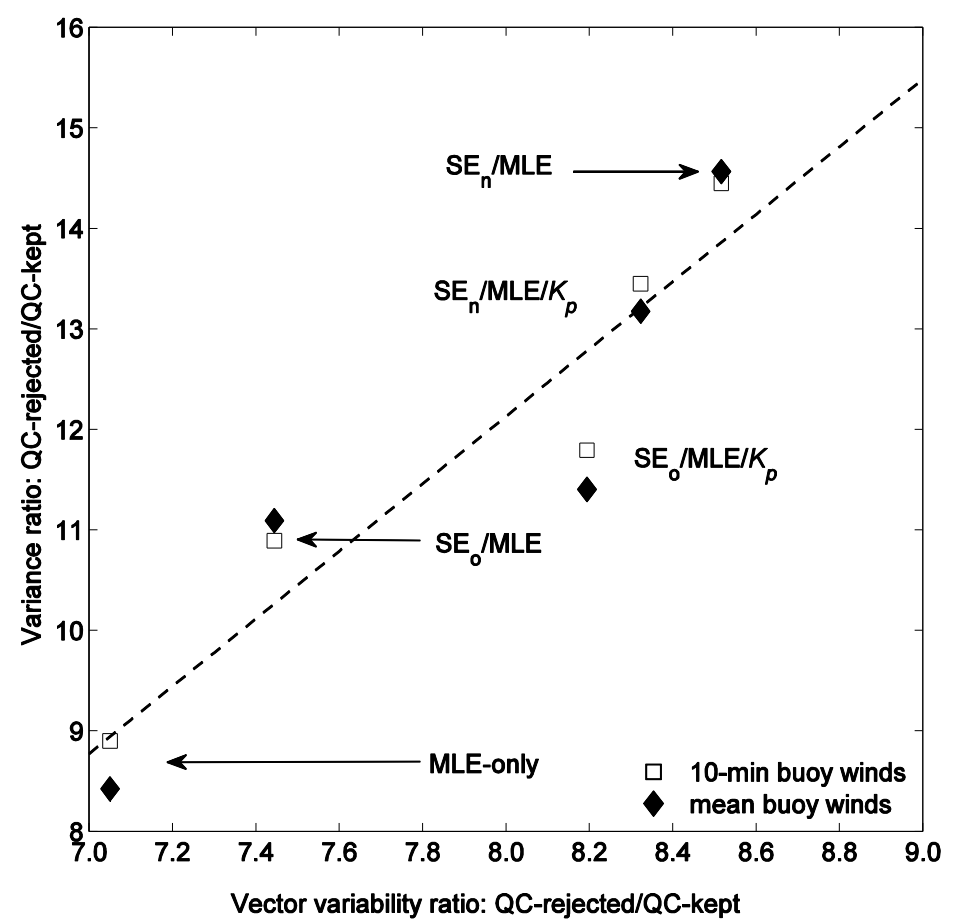

Fig. 3 The variance ratio (w.r.t. buoy winds) between QC-rejected and QC-kept winds as a function of the buoy wind variability ratio in these two categories.

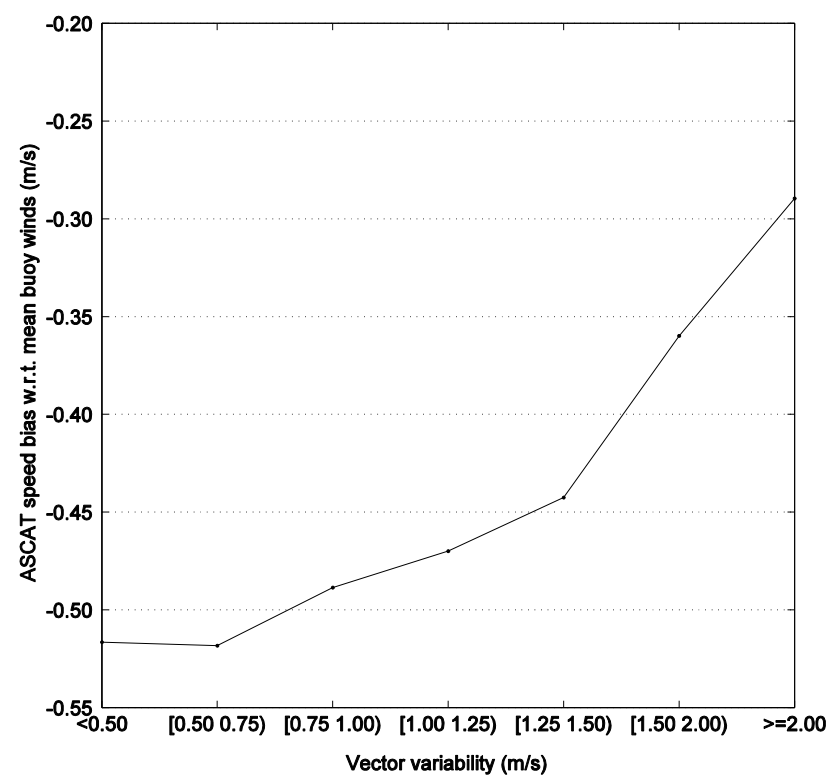

Fig. 4 ASCAT wind speed bias w.r.t. mean buoy winds as a function of the categorized wind vector variability $S D_{\text {vector }}$ at low wind conditions. 